\title{
Women and Sports: View from Stereotyping \& Gender Differentials Perspectives
}

\author{
Md. Mahfuzur Rahman Khan (D) $ه$
}

Institute of Social Welfare \& Research, University of Dhaka, Bangladesh

$\triangle$ Corresponding Author: Md. Mahfuzur Rahman Khan, E-mail: mahfuzur.rkm@gmail.com

\author{
ARTICLE INFORMATION \\ Received: January 02, 2021 \\ Accepted: February 03, 2021 \\ Volume: 1 \\ Issue: 1 \\ DOI: 10.32996/jspes.2021.1.1.1
}

\section{KEYWORDS}

Women in Sports, Gender

Stereotyping, Gender Differentials

\section{ABSTRACT}

Sports are viewed as manly demesne, and this generalization brings about men seeing more noteworthy capacity and appending more prominent significance to the sport than women. Society foresees that men and women ought to get, have confidence in, and satisfy explicit gender differentials and stereotyping that have been set up. At the point when sexual orientation standards are disregarded, it is basic for names to be offered, inquiries to be posed, and individuals to be mocked. While "customary" gender stereotyping has remained genuinely consistent in the course of recent hundreds of years, they have likewise been tested and defied by numerous women and women's activists. One explicit region in which customary gender differentials have been assessed and dissected is sports and proactive tasks. In any creating field, for instance, the one that began as "women in sport," key advancements can be followed through the development of the language utilized and the ideas created to communicate new understandings. The current investigation has been attempted to target evaluating the contribution of women in sports in various fields. From the quantity of variables that impact women in sports, gender differentials and stereotyping have been chosen for this investigation. This investigation has uncovered that when correlations were made with their male partners, women athletes have been similarly solid and were discovered to be similarly skilled, inventive, creative and capable.

\section{Introduction}

In this day and age, men are required to be strong, free, and athletic, while women are depended upon to hush up, respectful, alluring nurturers. There are the general points have sorted out by some traditional researcher that how much variant biological and psychological differences between men and women which also effect on all that punk. Gender equality should be the social norm, which means people should be treated by society as a person. Because of generalizations are always and exclusively negative. People are not protons, electrons, or neutrons in that every person is the same, and that somehow there are a baseline. Now a days maximum women are not interested to sports, when everyone tells you non-stop that women are weak-minded, even if the women you know are evidently not, all though you might feel they are exceptions and still accept that women are week-minded and only men are fit as an athletic and day by day gender inequality has become social culture. Demographic, epidemiological, technological, and socio-economic transitions have involved to identify the nature of women's physical and psychological health. Climate, migration, rapid urbanization, greater economic participation, and humanitarian crisis are participating new risk factors for women and underlying gender inequalities in different ways. The 50 years since women have seen dramatic changes globally. Women are being well educated, they are going to abroad for higher study. Marinating political stage, working as a co-worker in various international organizations, participating in various sports and successfully comeback with award. In last 50 years, undoubtedly women sport has changed hugely, but in 1896 women were not allowed to compete in the first Olympics. Now media covers women's sports more and role models are essential part of getting kids into sport from a young age. All though still women are suffering from discrimination in workplace like wage, maternity and pregnancy leave but women survive this disparities field by their won mental strengthens. Gender inequality in the eyes of biology it's the identification of gender. It means, when someone unfairly judges a person based on their sex or gender. It is when he or she as a representative of a society belittles, insults, treats someone unfavorably due to their gender as well as gives preferential

K C AL-KINDI CENTER $\mathbf{R}$ D FOR RESEARCH AND R D DEVELOPMENT Your gatemay to world-class research

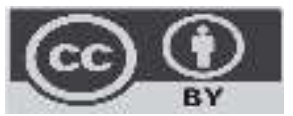

Published by Al-Kindi Center for Research and Development. Copyright (c) the author(s). This open access article is distributed under a Creative Commons Attribution (CC-BY) 4.0 license 
treatment someone just because on their gender. This Unfinished agenda highlights the lack of real political commitment to gender equality, as well as the power of the conservative backlash. Gender stereotyping is an interaction wherein youngsters' organic sex decides the exercises they take part in, just as the way in which they are treated in these exercises. Gender differentials are contrasts in the psychological capacities and practices of the genders, and are because of a mind boggling transaction of organic, formative, and social components. This contributes to the gender differences observed in sport.

\section{Theoretical Framework}

'Sport is a social movement where people can be presented to various sorts of segregation and social rejection, e.g., sex imbalance, homophobia, or potentially sexism (Cleland, 2014)' and 'it is a context wherein such conduct is frequently manifest and, in situations of comparison or division of gender, can give rise to direct conflict (Naranjo et al., 2017a, 2017b)'. Gender generalizing alludes to the act of crediting to an individual lady or man explicit ascribes, attributes, or parts by reason just of her or his participation in the social gathering of women or men. Gender discrimination is an unequal treatment between two persons on the base of race, gender, or ethnicity. Basically women's are the target of gender discrimination because according to society all powers are reserved for man not for woman. Men have all the rights over woman. Man can do whatever they want to do while woman can only do that a man wants from her. A woman does all the things according to the needs of man. She has no right to think about her own self. She has no right to take decisions about her marriage, about her education, about her career about her child, she is bound in all aspects of life. Women's are also suppressed on the name of religion. Literally Discrimination is an unequal treatment between two things. One is considered more powerful and another one is considered as more weak. Gender inequality is the type of disparities which is based on the gender of the person. At the same time, the understanding of sport has created from believing it to be a static social establishment, described similar to its division from this current reality, to the enthusiasm for sport as a social practice made through human office and copied through philosophical work. Gender differentials are differences among people that depend on organic transformations that are the equivalent for both genders. Gender is normally used to mean an organic distinction like characteristics that are explicitly dimorphic (diverse among people) though gender is all the more a reach and can incorporate various credits as a result of the social and social effects on general conduct. This have been advanced from considering gender orientation to be a variable or as a distributive class to thinking about it as a bunch of relations made through human relations and supported or imitated through social chips away at including, yet not restricted to, sport. Discriminating against someone because of their gender identity. It could be denying access to housing, refusing access to education, refusing to hire a person. Finally, the point of view on gender direction relations has moved from a consideration on gender contrasts, considered as tolerably characteristic, to a stunned response to genderism, to a more significant cognizance of precisely how multifaceted and socially orchestrated are the relations of control and coercion that depict gender relations in male centric societies.

\section{Methodology}

This paper is constructed by the utilizing research technique for systematic analysis of literatures. Methodical investigation of logical literature uncovered that gender stereotype \& gender differentials are critical while examining about women in sports. The current examination has been embraced targeting surveying the inclusion of women in sports in various fields. From the quantity of variables that impact women in sports, gender stereotype \& gender differentials have been chosen for this investigation. This study has uncovered that when correlations were made with their male partners, women athletes have been similarly solid and were discovered to be similarly proficient, inventive, creative and skilled.

\section{Research Gap}

There is a shortage of examination zeroing in on game investment from the perspectives of enthusiastic norm and sexual orientation differentials and generalization. Furthermore, just restricted exploration researched the connection between sport backing and individuals' character ascribes, for instance, fear of negative appraisal. As a result, the current study attempts to examine the influence of peer attitude, fear of negative evaluation, and gender differentials and stereotype on sport participation.

\section{Literature Review}

'Women have been required to wear dresses, cook, and clean, bring up kids, keep a lovely and sensitive body, and stay latent, moral, and unadulterated (Griffin, 1998)'. Women have been classified as women in regard of being judged as 'more vulnerable gender' - intellectually, truly and inwardly. If the scale of womanliness is applied, women are dependent on satisfying some particular jobs that are held by the two people in standard society. Women are being prevented from lifting loads, snorting, being forceful, partaking, and contending in sports and proactive tasks depending on this superficial prediction of feminism. "When women do "go too far" and display these supposed "masculine attributes", their gender personality, sexual direction, qualities, and social jobs are regularly addressed' (Griffin, 1998)'. Occasionally, women engaged in sports are the victim of Negative marks of disgrace that is falsely imposed upon them and thus is used as an instrument to control and restrict women 
participation in sports. This is also significant to ensure the authorization of gender roles in social perspectives where any individual would introduce themselves and are classified as women or men. 'Butler (2011) stated that people regard gender as a procedure or discourse whereas performative act in the meantime has gained popularity in the literature arena.' It is clearly evident that irrespective of issues, gender is not just a mere issue for mainstream researchers.

\section{Stereotyping and Gender Differentials}

Sports help women to establish their own disposition. Sports not only just give physical and mental strength, but also give a way to attain self-sufficiency to break the social boundaries in order to carry on with a better and quality life. In addition, it makes young women more self-reliant and more grounded. Many of them were treated as untouchables, newbies were allowed only to partake in 'specially considered' types of activity and sport. It was treated as the norm for a long time - and it might in any case be swept away in today's liberal time. Only specific sorts of sport and exercises were accessible to women whereas, men were allowed to play every single one. These disparities bring up the issue concerning this destroying or potentially de-performance of gender contrasts in, just as outside, sport is whether a sign pointing towards another sexual discrimination. Gender stereotype $\&$ differentials in sport participation has been a social regular phenomenon for decades. An explanation for gender stereotype indicates the strong link between sport behavior and masculinity. For viz, sport participation has been sorted as a masculine activity; literature even argues that participation in competitive sports diminishes the traditional gender roles of women. In sport, the physique, its introduction and its accomplishments, are given sense and importance through insight, understanding and assessment, whereas the points, the guidelines and the assessments of donning exercises rely on social plans. Diverse examinations, be it the correlation of various sports or be it an overview of the past, clearly reveal that it is simultaneously both the development and the implications related with it - along with the developments of femininity and masculinity as well as the structural and symbolic gender arrangements in a particular society - have higher probabilities to label the sporting activities as either male or women.

'Dividing sports along the lines of masculinity and femininity could encourage women to accept physical limits that have been placed on them (McClung et al, 2002)'. Findings also indicate that women's participation and experience in sports are influenced by gender stereotypes. Studies demonstrate that although society is more aware of gender stereotyping in sports and physical activities, traditional gender stereotypes for women still persist in the society. Women athletes have a subordinate position in the sports world. So, perpetuating the idea that sports and athletics continue to be more appropriate in the masculine domain. This gender inequality can be paralleled to the patriarchal nature of both society and sport. However, it can also continue to be challenged by encouraging girls and women to be physically active, involved with sports at all levels, and the desire to confront traditional women stereotypes. In outline, it very well might expressed that regardless of whether permeable, lines of division have been created in the sports between the genders. From one perspective, there are not many types of sport today from which one of the two genders is officially banned, (for example, men from synchronized swimming). We were preoccupied with the idea that young women can't go outside of her own or get back after the night since they are dependable, yet when one engages herself with sports activities, it certainly would give her the power to do her things alone. Women in sport were defined as the 'other gender' since early beginning. In the light of past turns of events, which have all been towards opening up various sports for women, it appears to eliminate any limitations that actually exist for people in sports. On the other hand, people interested in various relaxing sports give impression of progressive momentum in various ways.

\section{Findings}

The findings of these study results, however, show that inconsistencies in the building of gender and concomitancy between conformity and resolution exist where, in spite of their 'man' sports, female athletes desire, or are supposed to, meet conformist women's expectations. The study of understanding and inequities in sports and sports in recent decades shows girls and women making strides in sport and fitness as well as physical activity. This is partially due to efforts to increase the participation of women in all sports. There will be discussion of whether women succeed in creating a particular form of daily sports activity according to women's attributes in traditional 'male' sports. Studies have shown that expectations of body and appearance and dominant perceptions of femininity shift dramatically as women join the traditional sport of men. This does not entail, however, precisely the breakdown of sexual dichotomies. There is also a trend towards a steady increase in success in female sports, which makes it more and more necessary to adapt strategies and tactics. While women play men's sports and conform themselves to the standards and values that exist, men's participation in physical activities of 'typical women' is insignificant. Sport was usually listed as male and female. This relie on natural sex contrasts and not really what sexual orientation might interpret. These variations have been reported across sports, cultures and ages far from being products of socialization and environment. The visual depiction of intrinsic biological gender differences between men and women is also apparent in sport. One thing that was very understandable in the models alluded to above is that the interest in women in sport at an expert level has grown in the last few years. Women are encouraged to take an interest in groups and individual occasions with frequent home-grown 
competitions organized by different wearing organizations. Sadly, and perhaps not surprisingly, the current demand for equality and inclusion, along with the general misconception of biological sex as a process of evolution, have created concerns and misperception about conventional gender usage in sport.

\section{Discussion and Conclusion}

Sports and athletic realm are linked to the masculine arena and the female athlete has a history of partiality. Traditionally, in relation and comparison to men, children, women and femininity have been identified. One point that has been evident from the aforementioned examples is that in subsequent years the acceptance for women's sports to take part in competitive sports has grown. This phenomenon has been met and challenged over the last few decades. Women have been encouraged to compete in both team sports and individual competitions with frequent domestic tournaments hosted by various sporting federations. Conventional women's stereotypes have also shifted and incorporated gradually. Women obviously can break away from conventional expectations but there is still much to be done. Girls and women have taken narrow, negative, and restrictive concepts and ideas to discourage them from engaging in athletics, sweat, displaying violence or competing, even in the description of women's physical strength and athletic skills. This is possibly going to happen, until girls and women avoid feeling they have to choose between women and sports. Sport is also a marked example of how certain facets of society are done. Whether nationalism, leadership, teamwork, ambition, or people's desire to do genuinely great deeds, sports are an example of how these aspects connect and appear on the world stage.

This illustrates, in many respects, the problems with the deterioration of gender in other aspects of life, such as discriminations, reforms and equal pay issues. Young women, women and compassion have traditionally differentiated themselves in relation and disparity between men and manhood. The manly field is related to athletics and the world of competition, and a culture exists of predisposition towards female competitors. This trend has been revised and validated in the last few decades. Young girls and women "handled" ideas and feelings, which were tight, negative and restrictive, that should prevent sport, sweat, and animosity and compete and that began to remember real strengths and sportiness in the sense of gentleness. Women should clearly break free of conventional assertions, even though they are yet to go. Sport gives us frequent visual reminders of how many aspects of society work out. Whether patriotism, initiative, teamwork, intensity or people's desire to do truly amazing things, sport is an encapsulation of how they interact and present themselves on a global stage. Conventional women's generalizations have subsequently modified and evolved steadily. This is likely to happen once young women have the impression that they have to choose between sport and compassion. Sport also provides perhaps the most clear visual representations between people with inherent natural sex contrasts. The willingness for balance and incorporation and the general false impression about natural sex as a transformative cycle has caused shock, though perhaps obviously, inquiries and disarrays into the usual use of gender stereotypes and differences in sports. Somehow, this also has the problems which could be apparent in the disintegration of sex classes in various everyday issues, such as detention facilities, changing areas and the issue of equivalent restitution.

Funding: This research received no external funding.

Acknowledgments: We acknowledge there was no external funding support; all authors contributed accordingly in every part of the paper.

Conflicts of Interest: The authors declare no conflict of interest

\section{References}

[1] Butler, J. (2011). Gender trouble: Feminism and the subversion of identity. Routledge.

[2] Cleland, J. (2014). Racism, football fans, and online message boards: How social media has added a new dimension to racist discourse in English football. Journal of Sport and Social Issues, 38(5), 415-431.

[3] Griffin, P. (1998). Strong women, deep closets: Lesbians and homophobia in sport. Human Kinetics Publishers.

[4] McClung, L. R., \& Blinde, E. M. (2002). Sensitivity to gender issues: Accounts of women intercollegiate athletes. International Sports Journal, 6(1), 117-133.

[5] Naranjo, M., Luque, J., de la Mata, J., Torres, A., Redondo, J. and Paul, J. (2017a). Analyzing bad conducts in 14-16 year-old boys and girls football. En Proceedings ISSP 14th World Congress Sport Psychology, Sevilla (477). Madrid: Dykinson.

[6] Naranjo, M., Luque, J., de la Mata, J., Torres, A., Redondo, J. and Paul, J. (2017b). Intervention procedure and protocol to reduce the number of incidents in boys and girls football matches between the ages of 14 and 16 years old. En Proceedings ISSP 14th World Congress Sport Psychology, Sevilla (477-478). Madrid: Dykinson.

[7] Sherrow, V. (1996). Encyclopedia of Women and Sports. ABC-CLIO. Inc.: California. 